\title{
Reinforcement of metal with liquid-exfoliated inorganic nano-platelets
}

Peter May, Umar Khan and Jonathan N Coleman

School of Physics, CRANN and AMBER, Trinity College Dublin, Dublin 2, Ireland

*colemaj@tcd.ie

\begin{abstract}
We have prepared metal matrix composites (MMCs) of a pewter alloy filled with liquid-exfoliated Molybdenum Telluride $\left(\mathrm{MoTe}_{2}\right)$ nano-platelets. The combination of $\mathrm{MoTe}_{2}$ and pewter was chosen due to their near-identical densities, thus reducing the scope for buoyancy-induced separation during melt mixing. The addition of nanofiller results in a doubling of the Young's modulus, Y, for a volume fraction, $\mathrm{V}_{\mathrm{f}}$, of $<1 \% \mathrm{MoTe}_{2}$, corresponding to a reinforcement of $\mathrm{dY} / \mathrm{dV}_{\mathrm{f}}=110 \mathrm{GPa}$. We find that this degree of reinforcement to be reasonably consistent with that predicted by a simplified version of Halpin-Tsai theory.
\end{abstract}

Over the last decade, the study of 2-dimensional (2D) nano-materials has become one of the most active areas of nanoscience. Since the discovery of graphene ${ }^{1}$ and more recently, the surge of interest in inorganic 2D materials, ${ }^{2-4}$ such nanostructures have been used to demonstrate a host of applications in areas from composites to energy storage to optoelectronics. $^{2-5}$ Particularly important will be applications which harness the impressive mechanical properties of 2D nano-materials. It is well known that graphene is the strongest, stiffest material known to man with strength and elastic modulus values of 130 and $1000 \mathrm{GPa}$ respectively. ${ }^{6}$ Indeed graphene nanosheets have been used in a number of mechanical applications particularly as a reinforcing filler in composites. ${ }^{7-9}$ Less well known is the fact that boron nitride $(\mathrm{BN})$ nanosheets display very similar mechanical properties to graphene. ${ }^{10}$ Indeed a host of 2D nano-materials have Young's moduli which surpass $100 \mathrm{GPa} .{ }^{11}$ Perhaps more importantly these materials may have strengths ${ }^{11}$ as high as $36 \mathrm{GPa}$ (estimated for layered $\mathrm{WO}_{2}$ assuming its strength, $\sigma_{\mathrm{B}}$, and modulus, $\mathrm{Y}$, scale in a manner similar to $\mathrm{MoS}_{2}$ : $\left.\mathrm{Y}=11.7 \sigma_{\mathrm{B}}\right)^{12}$. However, to the author's knowledge only three papers describe using inorganic 2D materials to mechanically reinforce any matrices; in all cases reinforcement of polymer matrices with $\mathrm{BN}$ nanosheets. ${ }^{13-15}$

In many ways this is surprising. It was recently shown that a range of layered compounds can be exfoliated by sonication in liquids to give large quantities of 2D nanosheets. ${ }^{16-19}$ The advantage of such liquid processing is that the resultant suspensions can be used to make a range of materials and structures including composites. ${ }^{3}$ Because of the exceptional mechanical properties of $2 \mathrm{D}$ materials, ${ }^{11}$ we would expect such composites to display enhanced mechanical properties.

The vast majority of reports on nano-composites describe using nano-materials to reinforce polymer matrices. ${ }^{8}$ Yet, many other types of composites exist; including for 
example composite mixtures of two types of nanomaterial. ${ }^{20-22}$ One particularly interesting type of nano-composite which is of great technological importance is the metal matrix composite (MMC). These composites employ filler materials to improve the thermal, electrical and mechanical properties of metals, often aluminium and magnesium. Of nanomaterial-reinforced MMCs, much attention has been devoted to using carbon nanotubes (CNTs) to reinforce metals. ${ }^{23-36}$ Additionally, Boron Nitride nanotubes have been used to reinforce aluminium. ${ }^{37}$ Only recently have reports surfaced where two dimensional filler materials have been utilised in MMCs. In each case the filler was graphene ${ }^{38}$ or graphene oxide $^{9,24}$ while the matrix was aluminium. Impressive results were achieved with a neardoubling of tensile strength observed in one case. ${ }^{9}$

However, there is reason to believe that inorganic 2D materials may offer some advantages as fillers in MMCs. Several accounts from the literature highlight the presence of aggregation of CNTs either on the metal surface ${ }^{30-32}$ or within fissures in the metal matrix ${ }^{35}$. Such agglomerations may arise due to large density differences between filler and matrix phases within the composite resulting in buoyancy-induced separation during mixing in the molten state. ${ }^{39}$ This will be a particular problem when attempting to disperse low density materials such as CNTs (densities $<2000 \mathrm{~kg} / \mathrm{m}^{3}$ ) in denser metals such as aluminium or titanium (2700 and $4500 \mathrm{~kg} / \mathrm{m}^{3}$ respectively). Layered materials, on the other hand, have a range of densities from $\sim 2200 \mathrm{~kg} / \mathrm{m}^{3}$ for $\mathrm{BN}$ to $\sim 9400$ for $\mathrm{WTe}_{2}$, allowing the matching of filler density to that of the matrix thus reducing the scope for demixing.

Here we demonstrate that liquid-exfoliated 2D materials, specifically $\mathrm{MoTe}_{2}$ can be dispersed in a metal matrix, leading to mechanical reinforcement. In this proof of concept study, we use a low-melting point metal alloy, pewter, to facilitate melt mixing. The specific choice of $2 \mathrm{D}$ filler/metal combination was made such that their densities were within $5 \%$ of each other. While the dispersion was not perfect, it was reasonably good, leading to a doubling of stiffness relative to the metal itself.

The first stage in the production of the pewter/MoTe 2 composites studied in this work is the exfoliation of $\mathrm{MoTe}_{2}$ layered crystals to give exfoliated $\mathrm{MoTe}_{2}$ nano-platelets. Powdered $\mathrm{MoTe}_{2}$ (www.materion.com, M-1105, Density $7700 \mathrm{~kg} / \mathrm{m}^{3}$, particle size $<10 \mu \mathrm{m}$ ) was added to $80 \mathrm{ml} \mathrm{N}$-methylpyrrolidone (NMP) at a concentration of $15 \mathrm{mg} / \mathrm{ml}$. This mixture was sonicated overnight (15 hours) with a sonic tip (Sonics and Materials Inc, GEX600 at $25 \%$ of $600 \mathrm{~W}$ using a flat head probe). A pulsed setting ( $5 \mathrm{~s}$ on, $5 \mathrm{~s}$ off) was used to minimise solvent heating. To maximise the concentration, the dispersion was not centrifuged. For these samples, centrifugation was not absolutely necessary as the small size of the $\mathrm{MoTe}_{2}$ particles means no large unexfoliated crystallites remain after sonication. A few $\mathrm{ml}$ of the resulting dispersion was dropped onto a holey carbon grid (400 mesh) and analysed using a Jeol 2100 Transmission Electron Microscope (TEM) operating at 200kV. The images showed partially exfoliated nanosheets to be present in the dispersion, as shown in Fig 1A. These nanosheets were found to have an average length of $\langle L\rangle=620 \pm 470 \mathrm{~nm}$. For exfoliated nano-platelets such as these, it is difficult to accurately estimate the flake thickness. However, it is clear from the TEM images that while the $\mathrm{MoTe}_{2}$ has been exfoliated to some degree, the platelets 
still consist of a number of stacked flakes and so have thicknesses which are probably at least a few $\mathrm{nm}$. This dispersion was then filtered using a $0.45 \mu \mathrm{m}$ pore size nylon membrane to form a powder of weakly bound nano-platelets. ${ }^{40}$

For this work we choose a low-melting point metal to facilitate melt-mixing of the metal and nanosheets. We identified a high-grade lead-free pewter, usually used for homecasting of toy soldiers (www.princeaugust.ie, PA-2060, 94.5\% Tin / 3\% Zinc / 2.5\% Antimony - Melting Point $230^{\circ} \mathrm{C}$ - Density $7346 \mathrm{~kg} / \mathrm{m}^{3}$ ), as it could be easily melted on a hot plate. An as-received $80 \mathrm{~g}$ ingot of this metal is shown in Fig $1 \mathrm{~B}$.

A high $\mathrm{MoTe}_{2}$ content $(15 \mathrm{wt} \%)$ masterbatch was produced by first melting $8 \mathrm{~g}$ of pewter in a glass vial on a hot plate at $240^{\circ} \mathrm{C}$. When the metal reached a molten state, the required mass of $\mathrm{MoTe}_{2}$ powder was added and the mixture was stirred using a high speed rotor (a spatula blade attached to a power tool rotating at $15000 \mathrm{RPM}$ ) for 5 minutes. Once homogenised, the mixture was allowed to cool to form a powder. The degree of dispersion of nano-materials in metal matrices is usually controlled by a combination of the effectiveness of the mixing procedure, the degree with which the molten metal wets the nano-material surface and the density issues described above. ${ }^{39}$ Because of the density matching achieved here, we expect the degree of dispersion of the $\mathrm{MoTe}_{2}$ nano-platelets in the master batch to be limited by the crudeness of our mixing procedure as well as interfacial interactions at the pewter/MoTe 2 interface. Different mass fraction composites were made by adding the required amounts of masterbatch to already molten pewter in separate vials. Each sample was stirred at high speed and cooled to form a powder as before. The various composite powders were then placed onto a Kapton (melting point $~ 400 \mathrm{C}$ ) sheet and heated using the hot plate. When molten once more, the samples were pressed and cooled to form thin metal sheets. These were cut into strips of width $2.25 \mathrm{~mm}$ and average thickness $\sim 100 \mu \mathrm{m}$ using a die cutter.

To investigate the composition of the films, thin lamellae of both pewter-only and $1.5 \mathrm{wt} \%$ composite films were prepared using a Zeiss Orion Focused Ion Beam Microscope (FiB). Upon examination of the surfaces using a Zeiss Ultra Scanning Electron microscope (SEM), a number of islands were observed dotted on the surface (not shown). Elemental analysis (EDX) confirmed that these islands were Zinc, with Antimony and Tin present in the background. There was no difference in grain boundary or island concentration between the pewter and the composite. In addition, SEM was also used to examine fracture surfaces of the pewter-only and $1.5 \mathrm{wt} \%$ composite films. The fracture surface of the pewter only sample was relatively featureless, as shown in Fig 1D. However, for the composite film, clumps of aggregated platelets $(1-2 \mu \mathrm{m}$ in size) were observed uniformly distributed throughout the fracture surface as seen in Fig 1E. This suggests the $\mathrm{MoS}_{2}$ nano-platelets are not perfectly dispersed within the metal matrix. However, some regions of fracture surface were found where non-aggregated platelet-like objects could be seen protruding from the surface (fig. 1F). EDX confirmed these objects to be $\mathrm{MoTe}_{2}$.

Mechanical measurements were performed on a piece of as-bought pewter, strips of pewter processed in the same way as the composites and composites of various mass 
fractions using a Zwick Roell tensile tester with a $100 \mathrm{~N}$ load cell at a strain rate of $5 \mathrm{~mm} / \mathrm{min}$. For each mass fraction 5-8 strips were tested and the results averaged. Representative stress strain curves are shown in Fig 2A. The stress-strain curves for the pewter samples are similar to those reported for both Tin alloys ${ }^{41}$ and Zinc-Antimony alloys ${ }^{42}$. However, processing tends to increase the tensile strength, reduce the strain at break while leaving the modulus largely unchanged. Analysis of the processed pewter stress-strain curves gave values of Young's modulus, tensile strength and strain at break of: $Y=0.97 \pm 0.37 \mathrm{GPa}, \sigma_{\mathrm{B}}=45 \pm 11 \mathrm{MPa}$ and $\varepsilon_{\mathrm{B}}=13 \pm 3 \%$ respectively. It is clear from the curves in figure $2 \mathrm{~A}$ that adding $\mathrm{MoTe}_{2}$ results in a noteworthy increase in modulus coupled with a significant decrease in strain at break.

The Young's modulus is plotted as a function of $\mathrm{MoTe}_{2}$ volume fraction in Fig 2B (the as-bought pewter modulus is shown for comparison). The modulus increases linearly $\left(\mathrm{dY} / \mathrm{dV}_{\mathrm{f}}=110 \pm 10 \mathrm{GPa}\right)$, reaching a maximum of $\mathrm{Y}=1.93 \pm 0.6 \mathrm{GPa}$ for a $\mathrm{MoTe}_{2}$ content of $1 \mathrm{vol} \%$, before falling off at higher $\mathrm{MoTe}_{2}$ contents, probably due to aggregation effects. The maximum value corresponds to a doubling in modulus relative to both as-bought and processed pewter. To the best of our knowledge this is the first report of a modulus increase for MMCs filled with 2D nano-materials.

It is worth considering whether such modulus increases are as-expected. We can analyse the data using the well-known Halpin-Tsai model for reinforcement of continuous matrices with in-plane aligned platelets. Such in-plane alignment has been observed for composites of both graphene and BN nanosheets in polymer matrices. ${ }^{7,13}$ The Halpin-Tsai equations describe the composite modulus, $\mathrm{Y}$, as a function of the filler volume fraction, $\mathrm{V}_{\mathrm{f}}$, the moduli of filler, $\mathrm{Y}_{\mathrm{F}}$, and matrix, $\mathrm{Y}_{\mathrm{M}}$, as well as the platelet aspect ratio, L/t. This model has been shown to describe composites of $\mathrm{BN}$ nanosheets in polymer matrices and some MMCs quite well. ${ }^{13,43}$ Within this model, the composite modulus is given by ${ }^{44}$

$Y=Y_{M}\left[\frac{1+2 V_{f} \eta L / t}{1-V_{f} \eta}\right]$

where

$\eta=\frac{Y_{F} / Y_{M}-1}{Y_{F} / Y_{M}+2 L / t}$

For composites where $Y_{F} / Y_{P}>>1$, as is generally the case, the second expression can be rewritten as

$\eta \approx\left[1+2 \frac{L / t}{Y_{F} / Y_{M}}\right]^{-1}$ 
By inspection, it can be seen that this expression limits the range of values for $\eta$ to $0 \leq \eta \leq 1$. As a result, and because most nano-composites have low filler content, we can make the approximation that $V_{f} \eta<<1$, allowing us to combine equations 1 and 3 in a simplified form:

$$
Y \approx Y_{M}+\frac{Y_{F} V_{f}}{\left[\frac{Y_{F} / Y_{M}}{2 L / t}+1\right]}
$$

This equation is useful because it predicts the rate of increase of modulus with volume fraction, $d Y / d V_{f}$, to depend on properties of matrix and filler in a simple way:

$$
\frac{d Y}{d V_{f}} \approx \frac{Y_{F}}{\left[\frac{Y_{F} / Y_{M}}{2 L / t}+1\right]}
$$

From figure $2 \mathrm{~B}$, we know that $d Y / d V_{f}=110 \pm 10 \mathrm{GPa}$ and $\mathrm{Y}_{\mathrm{M}} \approx 1 \mathrm{GPa}$. Thus, equation 5 defines a relationship between $\mathrm{Y}_{\mathrm{F}}$ and $\mathrm{L} / \mathrm{t}$ which must be fulfilled for the Halpin-Tsai equations to accurately describe these composites. As far as we are aware there are no direct experimental measurements of the Young's modulus of $\mathrm{MoTe}_{2}$ nano-platelets. However, a number of measurements have shown the stiffness of $\mathrm{MoS}_{2}$ nanosheets to be in the range 270-330 $\mathrm{GPa}^{12,45}$. In addition, theoretical calculations of the in-plane stiffness of a range of layered crystals show that (when corrected for differences in monolayer thickness) ${ }^{46}$ the modulus of $\mathrm{MoTe}_{2}$ is approximately $60 \%$ of that of $\mathrm{MoS}_{2}{ }^{11}$ This allows us to estimate the stiffness of $\mathrm{MoTe}_{2}$ nano-platelets to be in the range 160-200 GPa. Then using equation 5 with the measured values of $d Y / d V_{f}$ and $\mathrm{Y}_{\mathrm{M}}$ shows that, if the Halpin-Tsai model is applicable here, the mean nano-platelet aspect ratio should be in the range $100 \leq L / t \leq 160$. Given that the mean flake length was measured to be $620 \pm 470 \mathrm{~nm}$ and the thickness of an $\mathrm{MoTe}_{2}$ monolayer is $0.7 \mathrm{~nm},{ }^{46}$ this means the nano-platelets would have to consist of < 15 stacked monolayers on average for the Halpin-Tsai equations to be accurate. This seems reasonable. Solvent exfoliated graphene tends to consist of flakes with thickness in the range 1-5 monolayers. ${ }^{47}$ It is clear from the TEM images such as that in figure $1 \mathrm{~A}$ that these $\mathrm{MoTe}_{2}$ nano-platelets are relatively poorly exfoliated compared to graphene. However, it is certainly possible that they consist of $<15$ monolayers. We note that if the true mean thickness of the nano-platelets in this work is greater than 15 monolayers, then this implies that the mean aspect ratio is $<100$. This means that, for the Halpin-Tsai model to accurately describe the data, the modulus of the $\mathrm{MoTe}_{2}$ would have to be greater than $200 \mathrm{GPa}$. On the other hand, if the flakes turned out to be thicker than 15 monolayers, it could also mean the Halpin-Tsai does not apply here, resulting in larger than expected composite stiffness.

The tensile strength does not increase significantly compared to the processed pewter as $\mathrm{MoTe}_{2}$ is added (figure 2C). However, as the $\mathrm{MoTe}_{2}$ content is increased beyond $1 \%$ the strength falls off, reaching $\sim 10 \mathrm{MPa}$ for $6 \% \mathrm{MoTe}_{2}$. However, we note that the strength of all samples with $\mathrm{MoTe}_{2}$ content below 5\% had strength greater than the as-bought pewter. 
Previous studies have observed strength increases at relatively low loading level followed by a falloff at higher filler content for MMCs filled with both carbon nanotubes ${ }^{25,26,32}$ and graphene $^{24}$. A strength decrease, often at higher loading levels is commonly observed for nanomaterial-filled polymer composites. ${ }^{7,13,48}$ Such behaviour has been attributed to filler aggregation. ${ }^{49}$ As such, degradation of strength with $\mathrm{MoTe}_{2}$ content is to be expected given the platelet aggregation observed in Fig 1E. The strain at break also decreases relatively uniformly with increased levels of $\mathrm{MoTe}_{2}$. This behaviour has been observed for several other MMCs ${ }^{9,24,25,28,30,32,34}$ and indeed polymer nano-composite materials ${ }^{7,13,48,50-55}$.

In conclusion, we have demonstrated a method to prepare composites of exfoliated $\mathrm{MoTe}_{2}$ nano-platelets in a metal matrix. Use of a low melting point matrix such as pewter simplifies the process of melt mixing while matching filler and matrix densities reduced the propensity for buoyancy driven de-mixing. While such processing results in sub-optimal dispersion of the nano-platelets, some well-dispersed $\mathrm{MoTe}_{2}$ nanosheets were observed. We found the Young's modulus of a $1 \% \mathrm{MoTe}_{2}$ composite to be double that of the pewter itself. Such an increase agrees reasonably well with the predictions of theory. However, both strength and strain at break fell with increasing MoTe2 content, possibly due to aggregation effects.

We acknowledge the European Research Council for financial support via the grant SEMANTICS. 

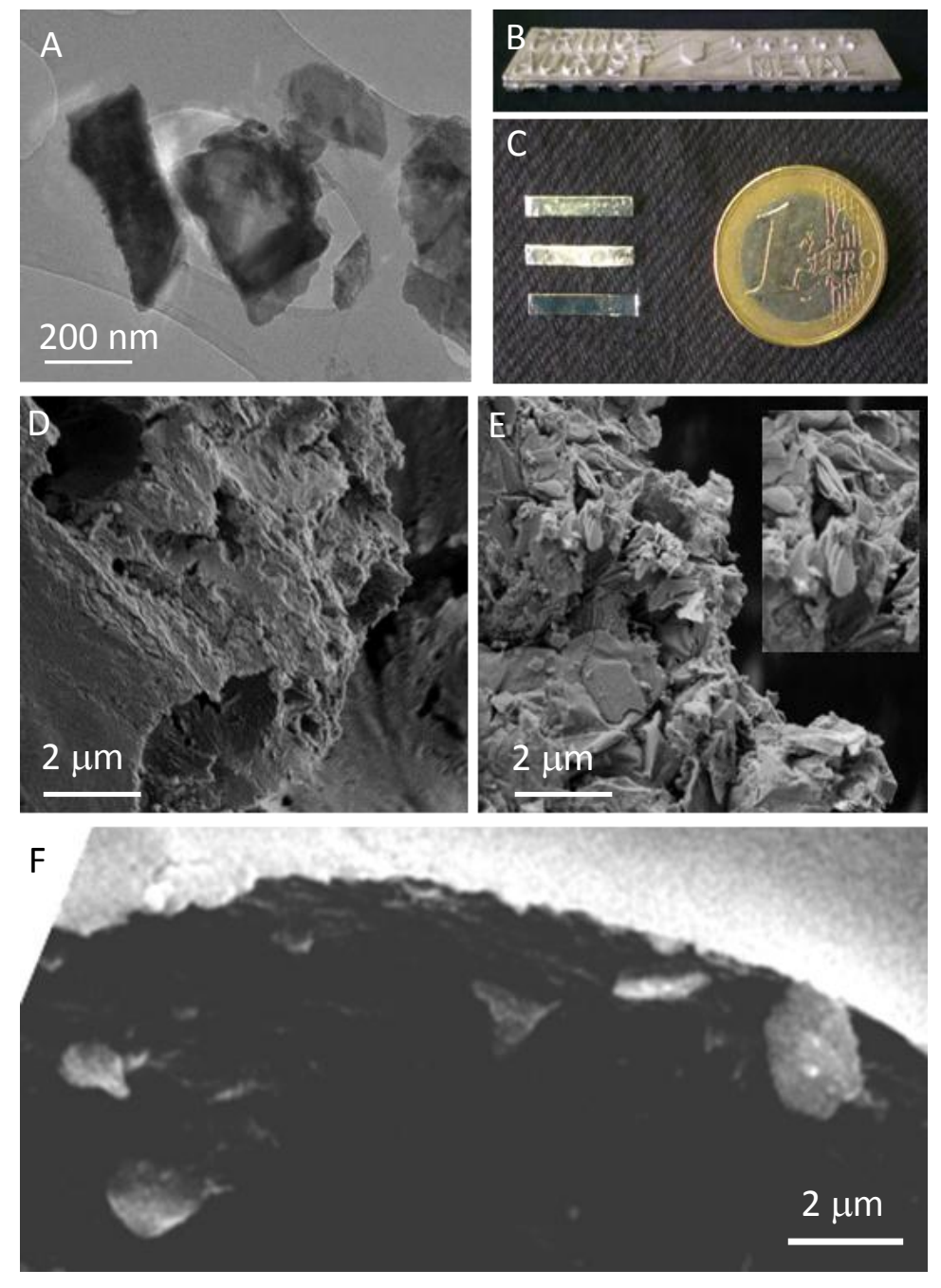

Figure 1: A) TEM images of $\mathrm{MoTe}_{2} / \mathrm{NMP}$ dispersion with many multilayer nanosheets present. B) Photo of an as-received pewter ingot. C) Pewter-only (top) and composite strips used for mechanical testing. D) SEM image of pewter fracture surface. E) SEM image of a $1.5 \mathrm{wt} \%$ composite fracture surface. F) SEM image of the fracture surface of a $1.5 \mathrm{wt} \%$ composite film with protruding nano-platelets visible. 

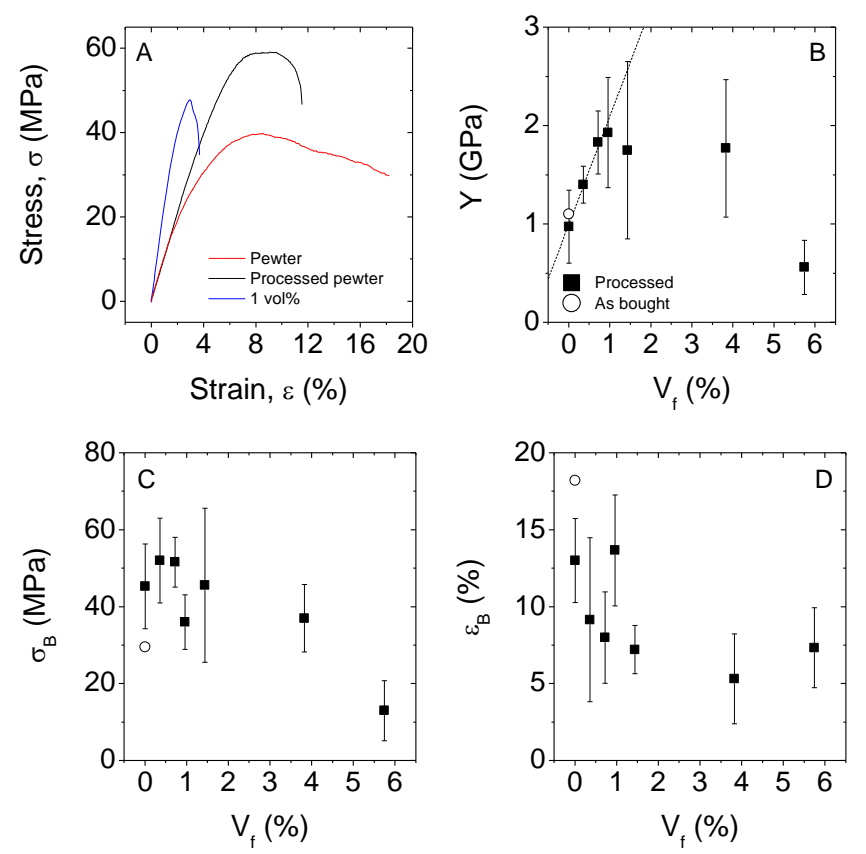

Figure 2: A) Representative stress-strain curves for a piece of as-bought pewter, a pewteronly film processed in the same manner as the composites and a composite film (1vol\%). BD) Plots of B) modulus, C) ultimate tensile strength and D) strain at break versus filler volume fraction for the processed pewter and composite films. For comparison the properties of as-bought pewter are also shown. The dashed line in B represents a slope of $125 \mathrm{GPa}$.

\section{References}

A. K. Geim, Science 324, 1530 (2009).

M. Chhowalla, H. S. Shin, G. Eda, L. J. Li, K. P. Loh, and H. Zhang, Nat. Chem. 5, 263 (2013).

V. Nicolosi, M. Chhowalla, M.G. Kanatzidis, M.S. Strano, and J.N. Coleman, Science 340, 1226419 (2013).

Q. H. Wang, K. Kalantar-Zadeh, A. Kis, J. N. Coleman, and M. S. Strano, Nature Nanotechnology 7, 699 (2012).

K. S. Novoselov, V. I. Fal'ko, L. Colombo, P. R. Gellert, M. G. Schwab, and K. Kim, Nature 490, 192 (2012).

C. Lee, X. D. Wei, J. W. Kysar, and J. Hone, Science 321, 385 (2008).

P. May, U. Khan, A. O'Neill, and J. N. Coleman, Journal of Materials Chemistry 22, 1278 (2012).

T. Kuilla, S. Bhadra, D. H. Yao, N. H. Kim, S. Bose, and J. H. Lee, Progress in Polymer Science 35, 1350 (2010).

9 J. Wang, Z. Li, G. Fan, H. Pan, Z. Chen, and D. Zhang, Scripta Materialia 66, 594 (2012).

10 L. Duclaux, B. Nysten, J. P. Issi, and A. W. Moore, Physical Review B 46, 3362 (1992).

11 C. Ataca, H. Sahin, and S. Ciraci, Journal of Physical Chemistry C 116, 8983 (2012).

12 S. Bertolazzi, J. Brivio, and A. Kis, ACS Nano 5, 9703 (2011).

13 U. Khan, P. May, A. O'Neill, A. P. Bell, E. Boussac, A. Martin, J. Semple, and J. N. Coleman, Nanoscale 5, 581 (2013).

Y. Wang, Z. X. Shi, and J. Yin, Journal of Materials Chemistry 21, 11371 (2011). 

2889 (2009).

J. N. Coleman, M. Lotya, A. O'Neill, S. D. Bergin, P. J. King, U. Khan, K. Young, A. Gaucher, S. De, R. J. Smith, I. V. Shvets, S. K. Arora, G. Stanton, H. Y. Kim, K. Lee, G. T. Kim, G. S. Duesberg, T. Hallam, J. J. Boland, J. J. Wang, J. F. Donegan, J. C. Grunlan, G. Moriarty, A. Shmeliov, R. J. Nicholls, J. M. Perkins, E. M. Grieveson, K. Theuwissen, D. W. McComb, P. D. Nellist, and V. Nicolosi, Science 331, 568 (2011).

G. Cunningham, M. Lotya, C.S. Cucinotta, S. Sanvito, S.D. Bergin, R. Menzel, M.S.P. Shaffer, and J.N. Coleman, Acs Nano 6, 3468 (2012).

A. O'Neill, U. Khan, and J. N. Coleman, Chem. Mat. 24, 2414 (2012).

R. J. Smith, P. J. King, M. Lotya, C. Wirtz, U. Khan, S. De, A. O'Neill, G. S. Duesberg, J. C. Grunlan, G. Moriarty, J. Chen, J. Z. Wang, A. I. Minett, V. Nicolosi, and J. N. Coleman, Advanced Materials 23, 3944 (2011).

G. Cunningham, M. Lotya, N. McEvoy, G. S. Duesberg, P. van der Schoot, and J. N. Coleman, Nanoscale 4, 6260 (2012).

P.J. King, U. Khan, M. Lotya, S. De, and J. N. Coleman, ACS Nano 4, 4238 (2010).

V. C. Tung, L. M. Chen, M. J. Allen, J. K. Wassei, K. Nelson, R. B. Kaner, and Y. Yang, Nano Letters 9, 1949 (2009).

S. R. Bakshi, D. Lahiri, and A. Agarwal, International Materials Reviews 55, 41 (2010).

S. F. Bartolucci, J. Paras, M.A. Rafiee, J. Rafiee, S. Lee, D. Kapoor, and N. Koratkar, Materials Science and Engineering: A 528, 7933 (2011).

C. F. Deng, D. Z. Wang, X. X. Zhang, and A. B. Li, Materials Science and Engineering: A 444, 138 (2007).

A. M. K. Esawi, K. Morsi, A. Sayed, A. Abdel Gawad, and P. Borah, Materials Science and Engineering: A 508, 167 (2009).

A. M. K. Esawi, K. Morsi, A. Sayed, M. Taher, and S. Lanka, Composites Science and Technology 70, 2237 (2010).

L. Jiang, Z. Li, G. Fan, L. Cao, and D. Zhang, Carbon 50, 1993 (2012).

L. Jiang, Z. Li, G. Fan, L. Cao, and D. Zhang, Scripta Materialia 66, 331 (2012).

H. Kwon, M. Estili, K. Takagi, T. Miyazaki, and A. Kawasaki, Carbon 47, 570 (2009).

J-Z. Liao, M-J. Tan, and I. Sridhar, Materials \& Design 31, S96 (2010).

Z. Y. Liu, B. L. Xiao, W. G. Wang, and Z. Y. Ma, Carbon 50, 1843 (2012).

R. Pérez-Bustamante, C. D. Gómez-Esparza, I. Estrada-Guel, M. Miki-Yoshida, L. LiceaJiménez, S. A. Pérez-García, and R. Martínez-Sánchez, Materials Science and Engineering: A 502, 159 (2009).

X. Yang, E. Liu, C. Shi, C. He, J. Li, N. Zhao, and K. Kondoh, Journal of Alloys and Compounds 563, 216 (2013).

D. K. Lim, T. Shibayanagi, and A. P. Gerlich, Materials Science and Engineering: A 507, 194 (2009).

C. S. Goh, J. Wei, L. C. Lee, and M. Gupta, Materials Science and Engineering: A 423, 153 (2006).

M. Yamaguchi, A. Pakdel, C. Y. Zhi, Y. Bando, D. M. Tang, K. Faerstein, D. Shtansky, and D. Golberg, Nanoscale Res. Lett. 8 (2013).

L-Y. Chen, H. Konishi, A. Fehrenbacher, C. Ma, J-Q. Xu, H. Choi, H-F. Xu, F. E. Pfefferkorn, and X-C. Li, Scripta Materialia 67, 29 (2012).

T. Noguchi, A. Magario, S. Fukazawa, S. Shimizu, J. Beppu, and M. Seki, Materials Transactions 45, 602 (2004).

U. Khan, H. Porwal, A. O’Neill, K. Nawaz, P. May, and J.N. Coleman, Langmuir 27, 9077 (2011).

T. Rameshkumar, I. Rajendran, and A. D. Latha, Tribology in Industry 32 (2010).

F. M. Azizan, H. Purwanto, and M.Y. Mustafa, International Journal of Engineering \& Technology 12, 78 (2012).

S. R. Bakshi and A. Agarwal, Carbon 49, 533 (2011).

R. J. Young, I. A. Kinloch, L. Gong, and K. S. Novoselov, Composites Science and Technology 72, 1459 (2012). 
A. Castellanos-Gomez, M. Poot, G. A. Steele, H. S. J. van der Zant, N. Agraït, and G. RubioBollinger, Advanced Materials 24, 772 (2012).

J. A. Wilson and A. D. Yoffe, Advances in Physics 18, 193 (1969).

U. Khan, A. O'Neill, M. Lotya, S. De, and J. N. Coleman, Small 6, 864 (2010).

H. Hu and G. Chen, Polymer Composites 31, 1770 (2010).

J. N. Coleman, U. Khan, W. J. Blau, and Y. K. Gun'ko, Carbon 44, 1624 (2006).

L. Jiang, X-P. Shen, J-L. Wu, and K-C. Shen, Journal of Applied Polymer Science, n/a (2010).

J. Liang, Y. Huang, L. Zhang, Y. Wang, Y. Ma, T. Guo, and Y. Chen, Advanced Functional Materials 19, 2297 (2009).

K. Nawaz, U. Khan, N. Ul-Haq, P. May, A. O’Neill, and J. N. Coleman, Carbon 50, 4489 (2012).

K. W. Putz, O. C. Compton, M. J. Palmeri, S-B. T. Nguyen, and L. C. Brinson, Advanced Functional Materials 20, 3322 (2010).

X. Yang, L. Li, S. Shang, and X-M. Tao, Polymer 51, 3431 (2010).

55

Xin Zhao, Qinghua Zhang, Dajun Chen, and Ping Lu, Macromolecules 43, 2357 (2010). 